Para enlazar con este artículo / To link to this article:

https://doi.org/10.6035/MonTI.2020.12.11

Para citar este artículo / To cite this article:

Serrat Roozen, Iris. (2020) "Accesibilidad audiovisual en la web: subtitulación en el Parlamento Europeo." En: Richart-Marset, Mabel \& Francesca Calamita (eds.) 2020. Traducción y Accesibilidad en los medios de comunicación: de la teoría a la práctica / Translation and Media Accessibility: from Theory to Practice. MonTI 12, pp. 313-344.

\title{
ACCESIBILIDAD AUDIOVISUAL EN LA WEB: SUBTITULACIÓN EN EL PARLAMENTO EUROPEO
}

\author{
IRIS SERRAT ROOZEN \\ iris.serrat@uv.es \\ Universidad de Valencia
}

\section{Resumen}

El presente trabajo pone de relevancia la importancia de la accesibilidad en la sociedad de la información actual, en concreto, en Internet, por lo que analizaremos el cumplimiento de los requisitos de accesibilidad a los contenidos audiovisuales establecidos por las Pautas de Accesibilidad al Contenido Web (WCAG) 2.0 en el canal de televisión online EuroparlTV. Asimismo, trazaremos el vínculo inherente entre la accesibilidad web y la audiovisual y describiremos los subtítulos generados por profesionales de la traducción en el ámbito institucional, concretamente en el Parlamento Europeo. Para llevar a cabo nuestro objetivo y poder realizar el estudio sistemático y exhaustivo de distintos aspectos propios de la subtitulación, hemos creado y analizado el Corpus EMPAC (EuroparlTV Multimedia Parallel Corpus) que reúne los subtítulos en inglés y en español de los vídeos emitidos en EuroparlTV entre 2009 y 2017.

Palabras clave: Accesibilidad; Traducción audiovisual; Subtitulación; Corpus.

\begin{abstract}
"Audiovisual accessibility on the web: subtitling in the European Parliament"

This study highlights the importance of accessibility in our current information society, particularly on the Internet. Therefore, we will analyse compliance with the requirements for accessibility to audiovisual content, established by the Web Content Accessibility Guidelines (WCAG) 2.0, on the online television channel EuroparITV. Furthermore, we will trace the inherent link between web and audiovisual
\end{abstract}


accessibility and describe the subtitles generated by translation professionals in the institutional sphere - in this case, at the European Parliament. To achieve our objective and conduct a systematic and exhaustive study of different aspects of subtitling, we have created and analysed the EMPAC Corpus (EuroparlTV Multimedia Parallel Corpus), which collates the English and Spanish subtitles of the videos broadcast on EuroparlTV between 2009 and 2017.

Keywords: Accessibility; Audiovisual translation; Subtitling; Corpus.

\section{Introducción}

La Declaración Universal de los Derechos Humanos, en el Artículo 27.1, afirma con claridad meridiana que toda persona, pertenezca a grupos mayoritarios o minoritarios de la sociedad, tiene derecho a "tomar parte libremente en la vida cultural de la comunidad, a gozar de las artes y a participar en el progreso científico y en los beneficios que de él resulten" (Asamblea General de la ONU, 1948).

La inclusión de todas las personas en la sociedad en la que habitamos está, irremediablemente, vinculada a la accesibilidad, al desarrollo tecnológico y, en concreto, a la posibilidad de usar Internet en igualdad de condiciones. De hecho, parece indiscutible que este medio ha supuesto un cambio vertiginoso en la organización social actual y ha generado profundas transformaciones en casi todas las esferas sociales. Hoy en día, Internet es una herramienta indispensable para trabajar, estudiar, hacer gestiones con la administración, divertirse, $y$, sin duda, es también un instrumento que puede fomentar una convivencia más inclusiva, heterogénea y justa. En consecuencia, no ha de sorprender que realizar tareas cotidianas, como navegar por Internet y consultar los contenidos textuales y audiovisuales que se muestran en este medio, sea un derecho reconocido por ley. Y, sin embargo, es este un derecho cuya plena ejecución, por el momento, creemos, es una asignatura pendiente, tanto en España como en otros países europeos.

Este trabajo pretende sumar esfuerzos en este sentido, poniendo de relieve la importancia de la accesibilidad y su vínculo indeleble con los Estudios de Traducción, en general, y la traducción audiovisual, en particular. El concepto de accesibilidad se ha colado en nuestra disciplina para quedarse y ocupar, a nuestro entender, un lugar central. 
Los avances tecnológicos e Internet han generado grandes cambios en la industria audiovisual y en la traducción audiovisual, tanto es así que, como apunta Orrego (2013), la velocidad de transferencia de datos y el almacenamiento, siempre en aumento, han convertido a Internet en el hábitat perfecto de los contenidos audiovisuales en detrimento de los contenidos puramente textuales. Por tanto, parece lógico recordar que para que un mayor número de usuarios pueda disfrutar de los vídeos alojados en la web, la subtitulación para personas sordas (SPS), la audiodescripción (AD) y la subtitulación convencional, entre otras modalidades de traducción audiovisual, son técnicas fundamentales que posibilitan el acceso al material publicado en la web. En referencia al tipo de información cada vez menos textual y más audiovisual hay autores que hablan de la web audiovisual, como es el caso de Bartolomé et al. (2007: 31)

En efecto, cada día es más natural la presencia de textos audiovisuales en Internet [...] Así pues, podríamos decir que el exponencial crecimiento de contenidos audiovisuales es la verdadera revolución en Internet en los dos últimos años, inaugurando el 'final del principio' de la Red de redes.

Por tanto, nos encontramos ante un escenario en el que la traducción audiovisual (TAV) en Internet debería ser central y la investigación en este ámbito sustancial. No obstante, si bien en los últimos años han proliferado los trabajos sobre la accesibilidad audiovisual en otros medios (Romero-Fresco 2019, Pedersen 2017, entre otros), el estudio de contenidos audiovisuales en Internet es, actualmente, terreno prácticamente ignoto.

Nosotros, con la intención de impulsar estudios en este ámbito, hemos analizado material alojado en la web del Parlamento Europeo, en concreto en el canal de televisión online EuroparlTV. Esta plataforma, que distribuye material audiovisual subtitulado en 24 lenguas, nos ha permitido, por una parte, estudiar la subtitulación online generada por profesionales de la traducción en el contexto institucional y, por otra, analizar el cumplimiento de los requisitos de accesibilidad de los contenidos audiovisuales establecidos por las WCAG 2.0, normativa desarrollada por la Iniciativa de Accesibilidad Web (WAI) del Consorcio World Wide Web (W3C). Con el objetivo de realizar un estudio de estas características hemos creado el corpus EMPAC (EuroparlTV 
Multimedia Parallel Corpus) que reúne los subtítulos en inglés y en español de los vídeos emitidos en EuroparlTVe entre 2009 y 2017.

Por otra parte, la traducción audiovisual en el contexto institucional nos ha parecido un marco de indiscutible valor para analizar el cumplimiento de los requisitos de accesibilidad establecidos por las WCAG 2.0 y describir la subtitulación profesional en Internet, lo cual posibilita la comparación con la subtitulación en otros canales (TV, cine, DVD, etc.). Como apunta Fuentes-Luque (2015), las instituciones u organizaciones públicas tienen la obligación de hacer que toda la información de sus webs sea accesible y estas consideran primordial el uso del material audiovisual. Del mismo modo, el autor las anima a trabajar de forma conjunta para establecer estándares comunes a la hora de implementar la TAV con el fin de asegurar la calidad y la accesibilidad del contenido.

Por su parte, Díaz Cintas considera necesario analizar el aumento de la velocidad de lectura de los subtítulos en distintos ámbitos (2012: 276):

The current state of affairs is one in which subtitling reading speeds tend to be considerably higher for the cinema and the DVD than for the television. The same can be applied to professional online subtitling, although, as a relative new comer, little research has been conducted in this field to verify it.

En definitiva, de un lado, Díaz Cintas anima al estudio de los aspectos técnicos de la subtitulación en Internet mientras que Fuentes-Luque invita a hacerlo en las webs de instituciones y organismos internacionales por el compromiso tácito que estas tienen con la accesibilidad y el derecho a la información de todas las personas. De la combinación de ambas propuestas nace nuestro estudio y el corpus EMPAC.

Nuestro trabajo persigue un objetivo doble, en primer lugar, describiremos en un análisis inicial, si los vídeos son accesibles, es decir, si cumplen con los criterios de conformidad establecidos por las WCAG 2.0. En segundo lugar, estudiaremos un aspecto esencial de la subtitulación y la SPS; la velocidad de lectura de los subtítulos.

1. Desde agosto de 2018 EuroparlTV pasó a ser el Multimedia Center, accesible en el siguiente enlace: https://multimedia.europarl.europa.eu/en/home?referer=\%22www. europarltv.europa.eu\%22 


\section{La accesibilidad en la sociedad de la información}

La relevancia del desarrollo tecnológico en nuestra sociedad es indiscutible. La forma de crear, tratar y distribuir la información ha cambiado profundamente y la velocidad que las tecnologías han imprimido al proceso en sí es abrumadora. De hecho, el termino sociedad de la información se refiere a una nueva forma de organización de la economía y de la sociedad caracterizada principalmente por el hecho de que casi cualquier ciudadano tiene, de forma instantánea, acceso ilimitado a la información.

En este sentido, Castells (2000) sostiene que, en realidad, Internet no es una tecnología, sino una nueva forma de organización de la producción y de los servicios operativos en la sociedad actual y en la economía. Por ende, es incuestionable la relevancia de Internet en casi todas las esferas de la vida y el valor del acceso a la información a través este medio. De hecho, entre los principios fundamentales de la Declaración de principios de la Cumbre Mundial sobre la Sociedad de la Información (CMSI 2004: 3) está el de "garantizar que las oportunidades que ofrecen las TIC redunden en beneficio de todos". Lógicamente para alcanzar este objetivo es necesario evitar la brecha digital asegurando que todos los ciudadanos tengan acceso a la información. Tenemos ante nosotros la oportunidad de aprovechar el desarrollo de las tecnologías de la información y la comunicación (TIC) con el fin de favorecer la inclusión en la sociedad actual.

Pero, ¿qué entendemos por brecha digital? Como apunta Valentí López (2002) el concepto se refiere al hecho de no poder disfrutar de las posibilidades que nos brindan las TIC de informarnos, comunicarnos, crear o aprender. Es una nueva forma de marginación que el autor denomina marginalización digital. Es importante destacar que la brecha digital se refiere a varias dimensiones como apunta Gómez Hernández et al. (2007: 31)

La brecha digital tiene dimensiones sociales, lingüísticas, educativas o culturales: Entre países o regiones con o sin acceso a la Red, entre jóvenes y mayores, entre medio rural y medio urbano, entre géneros, entre profesionales y clases trabajadoras, entre lenguas con presencia y contenidos en la Red o no, entre personas con discapacidades físicas, psíquicas o perceptivas que limitan el acceso y las que no las tienen, etcétera. 
El concepto se ha ensanchado hasta incluir cuestiones lingüísticas, de discapacidad y de alfabetización digital. En definitiva, una sociedad inclusiva se cimienta en el acceso igualitario a los sitios (movimiento), a la información (comunicación) y a los mensajes (comprensión); las barreras son la evidencia de la falta de acceso en alguno de estos tres ámbitos. Nuestro estudio abordará las barreras vinculadas a la comunicación, en concreto, las lingüísticas y aquellas que han de sortear las personas con algún tipo de discapacidad sensorial.

\section{Traducción audiovisual y accesibilidad}

Desde una aproximación dinámica y en consonancia con el objetivo de Delabastita (1989: 214) de superar el enfrentamiento terminológico entre traducción y adaptación, Mayoral (2001a: 46) propone que:

La definición del objeto de estudio de la traducción no es la definición de un proceso natural al que se le presupone inalterabilidad sino la definición de un proceso tecnológico que se encuentra en continua evolución y cambio. Nuestra función no es cerrar el paso a las nuevas realidades sino favorecerlas e impulsarlas.

Este enfoque da cabida a un nuevo concepto de gran relevancia en traducción audiovisual que no es otro que el de la accesibilidad. A nuestro entender, la traducción audiovisual se asienta en el binomio traducción y accesibilidad, siendo este segundo término intrínseco a la actividad que nos ocupa. Tanto es así que, como afirma Díaz Cintas (2009: 5), "Accessibility is a new key concept; an umbrella term that encompasses all associated new modes of translation". No es baladí, por tanto, subrayar que, en consonancia con la afirmación del autor, el término accesibilidad engloba y entrelaza varias modalidades de traducción.

Por su parte, Remael \& Neves (2007) indican que uno de los cambios más importantes se ha producido en el concepto mismo de la TAV. La transformación viene determinada por el cambio de rumbo al entender la TAV como una forma de accesibilidad "the fairly recent trend of defining audiovisual translation as a form of media accessibility" (Remael \& Neves (2007: 11), siendo esta la misma línea que recorren diversos autores (Díaz Cintas, Remael \& Orero 2007: 13) en traducción audiovisual. 
Accessibility is a form of translation and translation is a form of accessibility, uniting all population groups and ensuring that all cultural events, in the broadest sense of the word, can be enjoyed by all.

Pensamos que la esencia de la traducción es trasladar una pieza de información a las personas que quieran acceder a ella. En este sentido, Díaz Cintas (2007: 20) apunta:

Independientemente de que el obstáculo comunicativo sea de carácter lingüístico o sensorial, el objetivo del proceso traductor es exactamente el mismo: facilitar el acceso a una fuente de información y entretenimiento que de otra forma sería relativamente hermética.

El autor argumenta que, en esencia, el doblaje o el subtitulado de un contenido audiovisual tienen tanto de accesibilidad como la SPS y la AD. De hecho, si tenemos en cuenta que la mayor parte del contenido en Internet aparece en inglés, la barrera más importante es la lingüística.

\section{La accesibilidad web y las WCAG 2.0}

La World Wide Web tiene sus orígenes en el Centre Européen de Recherche Nucléaire (CERN) en Ginebra. En este centro de investigación, con el objetivo de crear un sistema de comunicación entre los científicos de física nuclear de todo el mundo, en 1991, Tim Berners-Lee combinó dos tecnologías existentes, el hipertexto y el protocolo de comunicaciones de Internet que dio lugar a la web. En la actualidad, este científico, lidera el World Wide Web Consortium (W3C), organismo internacional que promueve desde su creación en 1994 una web única cuyos beneficios estén disponibles para todos.

El trabajo del W3C se divide en cuatro pilares básicos: arquitectura, interacción, tecnología y sociedad, y accesibilidad web. Precisamente, este cuarto pilar propició la creación de la WAI, entidad que persigue la eliminación de barreras para las personas con discapacidad y la encargada de desarrollar las pautas de accesibilidad web para los diferentes componentes que la integran: Pautas de Accesibilidad para Herramientas de Autor (ATAG), Pautas de Accesibilidad para Herramientas de Usuario (UAAG) y Pautas de Accesibilidad al Contenido en la Web (WCAG). Todas son consideradas normas oficiales en la Unión Europea y se citan en la mayoría de las legislaciones sobre TIC de todo el mundo, en especial las WCAG que, por su 
repercusión y acogida, se han convertido en referente internacional aceptado y fueron aprobadas como estándar internacional ISO/IEC/ 40500:2012. Puesto que nuestro trabajo analiza la accesibilidad al contenido audiovisual en la web, nos centraremos en los aspectos más relevantes de las WCAG vinculados a nuestro ámbito de estudio, la traducción audiovisual.

En el siguiente apartado abordaremos en qué consiste la versión de las WCAG 2.0. Sin embargo, no queremos dejar de mencionar que existe una versión actualizada que sirve de transición hacia las futuras WCAG 3.0. No obstante, los criterios de conformidad que se ocupan de los contenidos audiovisuales no se han modificado en la versión 2.1 por lo que desarrollaremos aquí la versión 2.0, que siguen siendo el referente internacional aceptado.

\subsection{Pautas de accesibilidad al contenido en la web (WCAG) 2.0}

Las WCAG 2.0 se organizan en cuatro principios generales que establecen que el contenido ha de ser perceptible, operable, comprensible y robusto. Cada uno de estos principios recoge un conjunto de pautas (un total de 12) que describen los objetivos que se deben alcanzar para que el contenido sea accesible para todos. A su vez, cada pauta contiene una serie de criterios de conformidad (61 en total) que permiten comprobar si la página web es accesible. Por último, los niveles de conformidad que se asignan a cada criterio se clasifican en A (el nivel mínimo para que un sitio sea accesible), AA, AAA (el nivel más alto de accesibilidad). La normativa vigente indica que se tienen que cumplir todos los criterios con nivel de conformidad A y AA.

Los criterios de conformidad de las WCAG 2.0, a diferencia de las WCAG 1.0 , se han redactado para verificarse sin ambigüedad y son fundamentales para conseguir que una página web sea accesible. Con este objetivo en mente, la WAI ha generado varios documentos que facilitan la compresión y aplicación de cada uno de ellos: Understanding WCAG 2.0 y How to meet WCAG 2.0 que abordaremos más adelante.

Las WCAG 2.0, como comentábamos anteriormente, están estructuradas a partir de cuatro principios básicos. El primer principio, el de Perceptible ${ }^{2}$

2. http://www.sidar.org/traducciones/wcag20/es/. 
define cómo hacer el contenido audiovisual accesible a partir de las siguientes cuatro pautas:

1.1 Proporcione alternativas textuales para todo contenido no textual, de manera que pueda modificarse para ajustarse a las necesidades de las personas, como por ejemplo en una letra mayor, braille, voz, símbolos o un lenguaje más simple.

1.2 Proporcione alternativas sincronizadas para contenidos multimedia sincronizados dependientes del tiempo.

1.3 Cree contenidos que puedan presentarse de diversas maneras (como por ejemplo una composición más simple) sin perder la información ni su estructura.

1.4 Haga más fácil para los usuarios ver y oír el contenido, incluyendo la separación entre primer plano y fondo.

La Pauta 1.2 se ocupa de la accesibilidad al contenido audiovisual y comprende nueve criterios de conformidad, del 1.2.1 al 1.2.9. El criterio que indica que los vídeos deben incorporar subtitulación es el 1.2.2 y el 1.2.3 señala que estos deben también ir acompañados de audiodescripción. En el siguiente apartado abordaremos cómo se han de subtitular y audiodescribir los vídeos según las WCAG y que instrumentos proponen para la evaluación de la accesibilidad a estos contenidos.

\section{Cómo implementar y evaluar la subtitulación y la audiodescripión en la web}

Son varios los documentos que la WAI ha redactado para implementar y evaluar la accesibilidad de los contenidos en la web. El documento Understanding WCAG 2.0 explica el criterio en sí, los beneficios de alcanzarlo y una lista de ejemplos con los principales errores. Por su parte, How to meet WCAG 2.0 contiene información relativa a las técnicas que es aconsejable utilizar, y que de ser aplicadas garantizan que se cumpla el criterio de conformidad correspondiente.

La importancia del documento How to meet WCAG 2.0 reside en que alberga las técnicas que desde la WAI consideran adecuadas para cumplir con los criterios de conformidad. Las técnicas suficientes nos aseguran que, en caso de ser utilizadas, el criterio se alcanzará. Por su parte, las técnicas aconsejables mejoran la accesibilidad, si bien no aseguran la consecución de 
un criterio y, por último y de especial importancia, este documento facilita, por una parte, información sobre los fallos que provocan que no se cumpla el criterio de conformidad y, por otra parte, con el fin de facilitar la tarea a los evaluadores web, cada técnica propone un test que ayuda a determinar si la técnica se ha implementado con éxito. Muestra también ejemplos de cómo subtitular y audiodescribir para alcanzar el criterio y hace referencia a las guías de buenas prácticas que explicaremos en el siguiente apartado.

En el caso de la subtitulación (criterio 1.2.2), el test que nos permite evaluar si la página web satisface el criterio establece un sencillo protocolo de tres pasos: (1) visualizar todo el material con los subtítulos activados; (2) comprobar que todo diálogo va acompañado de subtítulos; (3) comprobar que toda la información sonora relevante está subtitulada.

En caso de no cumplir con el paso 2 o 3 no se alcanzaría el criterio de éxito y por tanto el contenido no sería accesible. Por otra parte, en cuanto a los fallos asociados a este criterio de éxito, encontramos que el fallo F8 3 indica lo siguiente:

NOTE: Captions sometimes simplify the spoken text both to make it easier to read and to avoid forcing the viewer to read at very high speed. This is standard procedure and does not invalidate a caption.

Vemos cómo se explicita que es recomendable evitar que el espectador se vea expuesto a velocidades de lectura demasiado rápidas lo que impediría la lectura cómoda de los mismos. Sin embargo, por lo demás, la información relativa a los parámetros técnicos de la subtitulación es absolutamente obviada en los criterios de éxito. Entendemos que esta no es una cuestión baladí puesto que son, en nuestra opinión, absolutamente centrales en la práctica de la subtitulación.

Parece evidente que los test que evalúan y determinan si un contenido audiovisual es accesible, se reducen a comprobar si el vídeo va acompañado de subtitulación o audiodescripción, pero no evalúan algunos aspectos esenciales de estas modalidades de traducción audiovisual. Puesto que nuestro estudio no solo determina, a partir de un análisis inicial, si los vídeos son accesibles según las WCAG 2.0, sino que también comprende el estudio de

3. https://www.w3.org/WAI/WCAG21/Techniques/failures/F8 
uno de los parámetros técnicos de la subtitulación, en el siguiente apartado presentaremos las normas que han servido de referente en nuestro análisis.

\section{Normas, guías y recomendaciones para la subtitulación on line}

Un código de buenas prácticas de subtitulación online sería muy beneficioso para todos los actores que participan en el proceso de desarrollo web, y en especial para los receptores de la información. No obstante, la realidad es otra y actualmente no hay una guía que estandarice estas prácticas en el entorno web, pero sí es cierto que, gracias a la redacción de normativas y leyes para alcanzar la igualdad de oportunidades encontramos guías y normas que definen y prescriben cómo se ha de subtitular.

Por otra parte, como indicábamos anteriormente, si bien la web no dispone de una normativa específica para la inclusión de los contenidos alternativos, las WCAG 2.0 sí hacen referencia a las recomendaciones del Described and Captioned Media Program (DCMP), organismo financiado por el Departamento de Educación de los EEUU y administrado por la Asociación Nacional de Sordos, que ha redactado las Caption Key (para SPS) y las Description Key (para AD). Tanto profesionales como amateurs de todo el mundo utilizan estas guías, redactadas para asegurar el acceso igualitario a la información.

Por su parte, España sí dispone de normativas específicas para SPS y AD. En el caso de la SPS encontramos la norma UNE 153010 publicada en 2003 y actualizada en 2012: "Subtitulado para personas sordas y personas con discapacidad auditiva". La actualización se realizó con el fin de adaptarse a las nuevas tecnologías y se considera norma general de subtitulado de aplicación para DVD, televisión general o Internet. La norma (2012: 5) explicita que:

Se contemplan todos los escenarios de comunicación audiovisual que permiten la incorporación del subtitulado y se consideran tanto el subtitulado en directo como el grabado, indicándose los casos en los que los requisitos no sean de aplicación común.

Igualmente, queremos resaltar el hecho de que en la redacción de la norma colaboraron asociaciones de personas sordas, empresas de subtitulado y medios audiovisuales, universidades y organismos estatales para así garantizar 
la homogeneidad e intentar establecer unos requisitos mínimos de calidad en la subtitulación para personas sordas.

Por otra parte, desde el ámbito profesional también se ha dotado a esta práctica de guías de estilo internas y recomendaciones que creemos imprescindible incluir en nuestro estudio. De un lado contemplaremos, por su larga experiencia y prestigio, las guías de estilo de la BBC (2009), institución que comenzó a subtitular a través del teletexto en 1979 y que en 2008 subtitulaba ya el $100 \%$ de su programación. De otro lado, con el afán de acercarnos a los contenidos audiovisuales que más se consumen, consideramos oportuno tener en cuenta las guías de subtitulación que Netflix ha creado para estandarizar la práctica de la subtitulación en todos sus contenidos. Conviene destacar que las guías no recogen diferentes velocidades de lectura según sea la subtitulación para personas con discapacidad auditiva o no, si bien sí recoge otros aspectos propios de la SPS. Por ejemplo, indica, entre otras recomendaciones, que la información sonora relevante tiene que ir entre corchetes. Netflix es una de las mayores plataformas de vídeo en streaming y un fenómeno mundial, por lo que merece ser considerada en un trabajo de estas características.

Por tanto, para analizar el parámetro velocidad de lectura, tomaremos como referencia tres documentos: la DCMP Captioning Key, la norma UNE 153010 de subtitulación y las BBC Subtitle Guidelines sin olvidarnos, en la reflexión teórica que acompaña a cada parámetro, de otras propuestas de calado académico y profesional.

\section{Análisis de la accesibilidad a los contenidos audiovisuales en la web}

El análisis inicial nos permite comprobar si el contenido audiovisual cumple con los criterios de éxito de las WCAG 2.0. El análisis se desarrolla en tres fases: una primera fase en la que determinamos si los vídeos van acompañados de subtitulación; una segunda fase en la que comprobamos si los subtítulos son convencionales o SPS; y una tercera en la que establecemos si los vídeos incorporan audiodescripciones.

En este análisis inicial, con el objetivo de determinar el grado de cumplimiento en materia de accesibilidad de los contenidos audiovisuales en la web, replicaremos el modelo de evaluación de Voces Merayo (2010), adaptándolo 
a nuestras necesidades para determinar si el material audiovisual alojado en EuroparlTV dispone de subtitulación, de SPS y de AD.

El modelo que presenta el autor (2010: 256) está compuesto por tres elementos que representan distintos niveles de concreción: módulos, parámetros e indicadores. Los módulos son dos: accesibilidad lógica del reproductor y accesibilidad del contenido audiovisual. Estos contienen parámetros que ocupan un segundo nivel de concreción y son los que definen qué se va a evaluar. En el caso del segundo módulo, accesibilidad del contenido audiovisual, plantea cuatro parámetros que coinciden con los cuatro principios generales que definen las WCAG 2.0: perceptible, operable, comprensible y robusto. Y finalmente, en el último nivel de concreción, se encuentran los indicadores que establecen cómo se evalúa cada parámetro de cada módulo.

En relación al parámetro perceptible propone tres indicadores: (1) subtitulación, (2) audiodescripción y (3) lengua de signos. La definición de los indicadores de este parámetro consiste en concretar si el contenido audiovisual incorpora subtitulación, audiodescripción o lengua de signos, y el procedimiento para verificarlo consiste en reproducir manualmente el contenido y constatar si se activa o no.

Por su parte, el parámetro comprensible contiene a su vez tres indicadores: (1) sigue las recomendaciones de audiodescripción UNE 153010, (2) sigue las recomendaciones de subtitulación UNE 153020 y (3) sigue las recomendaciones de lengua de signos UNE 139804. Sin embargo, consideramos que, por coherencia con las WCAG 2.0, los indicadores del parámetro comprensible se incluyen en el parámetro perceptible y suponen simplemente un nivel de concreción más dentro de ese indicador.

Seguidamente presentamos las tres fichas que hemos generado a partir de la metodología del autor (ampliada y adaptada), una para cada fase del estudio. En ellas se contemplan las propuestas de evaluación (test) vertidas desde la WAI alineadas con la propuesta de evaluación de contenidos audiovisuales de Voces Merayo (2010: 266-269) y en consonancia con nuestra metodología de análisis que nos permite automatizar el proceso y poder así analizar la gran cantidad de material que conforma nuestro corpus. 
Tabla 1: Ficha 1 para la evaluación de la accesibilidad audiovisual en EuroparlTV

\begin{tabular}{|l|l|}
\hline Definición & Evaluar si el contenido incorpora subtítulos. \\
\hline Examen & $\begin{array}{l}\text { Comprobar si existe subtitulación del contenido } \\
\text { audiovisual. }\end{array}$ \\
\hline Procedimiento & $\begin{array}{l}\text { Ejecutar un script que descarga todos los vídeos y registra } \\
\text { cuáles disponen de un archivo SRT con la subtitulación. }\end{array}$ \\
\hline Resultado & $\begin{array}{l}\text { El significado de los valores es: } \\
\text { SÍ I NO }\end{array}$ \\
\hline
\end{tabular}

Tabla 2: Ficha 2 para la evaluación de la accesibilidad audiovisual en EuroparlTV

\begin{tabular}{|l|l|}
\hline Definición & Evaluar si los subtítulos son convencionales o SPS. \\
\hline Examen & $\begin{array}{l}\text { Comprobar si los subtítulos incorporan información } \\
\text { sonora relevante. }\end{array}$ \\
\hline Procedimiento & $\begin{array}{l}\text { Analizar todos los archivos SRT y examinar si hay } \\
\text { información sonora relevante entre paréntesis o corchetes } \\
\text { con la herramienta CQPweb. } \\
\text { Examinar si hay información sonora relevante en } \\
\text { mayúsculas con CQPweb. }\end{array}$ \\
\hline Resultado & $\begin{array}{l}\text { El significado de los valores es: } \\
\text { SUBTITULACIÓN CONVENCIONAL: si los subtítulos } \\
\text { únicamente recogen la información textual. } \\
\text { SPS: si los subtítulos incorporan (entre paréntesis o } \\
\text { corchetes) información sonora relevante. }\end{array}$ \\
\hline
\end{tabular}

Tabla 3: Ficha 2 para la evaluación de la accesibilidad audiovisual en EuroparlTV

\begin{tabular}{|l|l|}
\hline Definición & Evaluar si el contenido incorpora audiodescripción. \\
\hline Examen & $\begin{array}{l}\text { Comprobar si existe audiodescripción del contenido } \\
\text { audiovisual. }\end{array}$ \\
\hline Procedimiento & $\begin{array}{l}\text { Examinar la web y el HTLM en busca de enlaces o } \\
\text { elementos de vídeo. }\end{array}$ \\
\hline Resultado & $\begin{array}{l}\text { El significado de los valores es: } \\
\text { SÍ I NO }\end{array}$ \\
\hline
\end{tabular}


Con esta metodología podremos determinar en qué medida los vídeos de EuroparlTV entre 2009 y 2017 son accesibles según las WCAG 2.0.

\section{Análisis del parámetro velocidad de lectura}

En la segunda parte de nuestro estudio analizamos el parámetro velocidad de lectura en los subtítulos de los vídeos del EuroparlTV. Este parámetro y las variables que hemos resuelto estudiar son, a nuestro entender, los que forman parte de la dimensión temporal (la espacial no la abordaremos en este análisis) propuesta por Díaz Cintas \& Remael (2007) siendo este un parámetro sobre el que se sustentan los principios básicos de la subtitulación. En este sentido, Díaz Cintas \& Remael (2007: 95) consideran que:

It is very frustrating and disconcerting to see how the subtitle disappears from the screen when we have not yet finished reading it, $[\ldots]$ the typical occasion in which we feel that we have 'read' rather than 'watched' the film.

Opinamos que, por muy elocuentes y ricas que sean las propuestas de traducción de los subtítulos, si no tenemos tiempo de leerlos, no podemos ni disfrutarlos ni tan siquiera, en ocasiones, entenderlos.

Los parámetros objeto de análisis en subtitulación y SPS han sido clasificados por distintos autores (Karamitrouglou, 1998; Díaz Cintas y Remael, 2007; Bartoll, 2008; Arnáiz, 2012; Tamayo, 2015, entre otros.) Nosotros partimos de la propuesta de Tamayo (2015a: 76) en la que presenta once parámetros y sus variables. Conviene recordar que la selección de las variables está cimentada en publicaciones previas de subtitulación convencional y SPS, y que reúnen las convenciones y características técnicas que han de tenerse en cuenta a la hora de crear e investigar en este ámbito.

Hemos considerado que el parámetro velocidad de lectura tenga tres variables, una más que la propuesta de Tamayo (ibíd.). A continuación, definiremos, por una parte, las variables objeto de estudio que presentamos en este artículo y, por otra parte, delimitaremos los valores que comprende cada variable a partir de una breve revisión teórica.

Sirva la siguiente tabla para resumir las variables de estudio y los valores correspondientes: 
Tabla 4: Resumen de las variables y valores de estudio

\begin{tabular}{|c|c|c|}
\hline PARÁMETRO & VARIABLE & VALORES POSIBLES \\
\hline \multirow{3}{*}{ Velocidad de lectura } & $\begin{array}{l}\text { Velocidad en caracteres } \\
\text { por segundo }\end{array}$ & $\begin{array}{l}15 \text { cps - Norma UNE y } \\
\text { DCMP } \\
16-20 \text { cps - Netflix } \\
\geq 21 \mathrm{cps}\end{array}$ \\
\hline & Pausa & $\begin{array}{l}0 \text { segundos } \\
\leq 0,16 \text { segundos } \\
>0,16 \text { segundos }\end{array}$ \\
\hline & Duración & $\begin{array}{l}\leq 1 \text { segundo } \\
1 \text { y } 6 \text { segundos } \\
>6 \text { segundos }\end{array}$ \\
\hline
\end{tabular}

\subsection{Variable velocidad de lectura en caracteres por segundo}

La variable velocidad de lectura en caracteres por segundo (cps) en nuestro estudio considerará tres valores: $15 \mathrm{cps}$, puesto que es la velocidad de lectura recogida en la norma UNE y en las guías DCMP, así como por ser la velocidad reconocida por la academia como la más habitual; un segundo valor que comprenderá todos aquellos subtítulos con velocidades de lectura entre 16 y $20 \mathrm{cps}$, que aunque exceden de la velocidad de lectura óptima recogida en la normativa, tanto la industria televisiva como las plataformas que ofrecen servicios online, como es el caso de Netflix, apuntan a valores que se mueven entre los 16 y los 20 cps; y por último, el grupo de los subtítulos imposibles, aquellos de 21 cps o más. En este sentido Pedersen (2017: 223) indica:

The time spent reading subtitles increases with reading speeds, so already at $15 \mathrm{cps}$, viewers spend on average about two thirds of their time in the subtitle area and at $16.5 \mathrm{cps}$, they spend $80 \%$ of their time reading subtitles. That may not be so much of a problem for relatively static genres such as interviews or news, but for a feature film, that leaves very little attention for the on-screen action.

El autor plantea, en su propuesta de análisis de la calidad de subtítulos, penalizar velocidades por encima de los $15 \mathrm{cps}$, si bien es cierto que la tendencia apunta a generar subtítulos entre 16 y 20 cps. Según Pedersen (2017: 223), $20 \mathrm{cps}$ equivale a unas $240 \mathrm{wpm}$ "which is a level where most people would probably do nothing else but reading subtitles (or stop using them)". Por lo 
que entiende que subtítulos con velocidades superiores a $20 \mathrm{cps}$ han de ser considerados como error en la subtitulación y no como una penalización.

\subsection{Variable pausa entre subtítulos}

La pausa entre subtítulos no es una variable que venga recogida en las guías que utilizamos como referencia en nuestro estudio. Sin embargo, teniendo en cuenta que son muchos los autores que la contemplan (Karamitroglou 1998; Ivarsson 1992; Castro Roig 2001; Mayoral 2001b; Díaz Cintas 2001; y González Iglesias 2012), incorporaremos su análisis en nuestro estudio.

En este sentido, Tamayo (2015: 280) matiza que no solo es importante la velocidad de los subtítulos, sino que también juegan un papel central las pausas entre estos. El ojo humano necesita tiempo para detectar la presencia de un nuevo subtítulo y cita a Sponholz (2003: 24):

At least four frames should be inserted between two consecutive subtitles in order to avoid the effect of a subtitle overlay. This time break is necessary to signal to the brain the disappearance of one subtitle and the appearance of another.

Por tanto, establecemos que la duración de la pausa entre subtítulos, en sintonía con el autor y otros investigadores prominentes en este campo (Ivarsson 1992; Castro Roig 2001; o Sponholz 2003; Tamayo 2015), tendrá un valor de 4 fotogramas $(0,16$ segundos) en nuestro estudio. Asimismo, creemos recomendable añadir dos valores que nos permitirán detectar si hay subtítulos que no dejan pausa entre ellos (pausa 0) y un tercer valor que recoja las pausas que superan los 0,16 segundos.

\subsection{Variable tiempo mínimo y tiempo máximo de permanencia en pantalla}

En nuestro estudio, la dimensión temporal añade esta variable que, aunque Tamayo no la ha incluido en este parámetro, consideramos necesario analizar. La famosa regla de los seis segundos habla por sí misma de la duración máxima de un subtítulo de dos líneas. Por su parte, Díaz Cintas \& Remael (2007: 89) suscriben esta norma, de igual manera que Koolstra et al., (2002) quienes pusieron a prueba la regla y concluyeron que seis segundos es el tiempo adecuado para que no se produzca una relectura del subtítulo. 
Por otra parte, también hay razones para establecer un tiempo mínimo de permanencia en pantalla, pues, como señalan Díaz Cintas \& Remael (2007), esto evita el efecto flash y garantiza que haya tiempo suficiente para leer el subtítulo. En este mismo sentido, Ivarsson \& Carrol (1998) indican que el tiempo mínimo de permanencia es de un segundo y Karamitroglou (1998) lo establece en 1 y 1/4 de segundo coincidiendo con la recomendación de la guía del DCMP. La norma UNE, sin embargo, no define este aspecto, de modo que, teniendo en cuenta que la norma comúnmente aceptada es de un segundo, tomaremos este valor para la variable tiempo mínimo de permanencia.

En suma, las variables y valores que planteamos aquí nos permiten describir si los subtítulos de nuestro corpus respetan las normas técnicas de la subtitulación teniendo en cuenta distintas realidades (académicas y profesionales), razón por la cual analizamos más de un valor para cada variable.

\section{Descripción y compilación del corpus EMPAC}

La web EUROPA es el portal oficial de la Unión Europea (UE) y a él se encuentran conectadas todas las páginas web de las instituciones y agencias europeas. La UE mantiene un compromiso con la accesibilidad, esta conexión implica que, de facto, las webs vinculadas a este portal se adhieren a su política de accesibilidad ${ }^{4} \mathrm{y}$ tienen que seguir las directrices internacionales de accesibilidad, incluidas las WCAG.

El corpus EMPAC se construye con la intención de analizar la subtitulación de vídeos emitidos en el canal de televisión online del Parlamento Europeo, EuroparlTV, y con el objetivo de estudiar su evolución a lo largo de los años. Es un corpus textual (puesto que no es representativo de una lengua), escrito, especializado (describe un sublenguaje), diacrónico y compuesto por textos completos que son del dominio público. Es a su vez un corpus multilingüe (contiene más de una lengua; en nuestro caso, inglés y español), paralelo y unidireccional (todas las traducciones son a partir de la misma lengua origen, el inglés) y alineado.

El número total de subtítulos de nuestro corpus asciende a 467.694 que aglutinan entre ambos 4.912.716 palabras; es decir, casi cinco millones de

4. https://europa.eu/european-union/abouteuropa/accessibility_es. 
palabras en medio millón de subtítulos. A continuación mostramos los datos de la versión del corpus en inglés (EMPAC_EN) y de la versión del corpus en español (EMPAC_ES):

Tabla 5: Datos de EMPAC_EN

\begin{tabular}{|c|c|c|c|}
\hline Textos & Subtítulos & Tokens (Componentes léxicos) & Duración \\
\hline 3812 & 224.036 & 2.448 .772 & 274 horas y $12 \mathrm{~min}$ \\
\hline
\end{tabular}

Tabla 6: Datos de EMPAC_ES

\begin{tabular}{|c|c|c|c|}
\hline Textos & Subtítulos & Tokens (Componentes léxicos) & Duración \\
\hline 3922 & 243.660 & 2.463 .944 & 283 horas y 2 min \\
\hline
\end{tabular}

El proceso de compilación del corpus EMPAC ha supuesto un reto considerable. Sin embargo, en este trabajo, no profundizaremos en el proceso de compilación dado que el objeto de nuestro análisis es otro. No obstante, sí queremos dejar constancia, de forma sucinta, de los pasos que hemos seguido y, de este modo, reflejar las posibilidades que brinda para futuros estudios.

\subsection{Compilación del corpus EMPAC}

A partir de procedimientos inspirados en la metodología del grupo de investigación European Comparable and Parallel Corpus of Parliamentary Speeches $(\mathrm{ECPC})^{5}$ de la Universitat Jaume I, los textos en formato digital que conforman nuestro corpus se han descargado de la web del Parlamento Europeo a partir de la información contenida en los HTLM. Una vez descargados todos los archivos con los subtítulos en SRT los transformamos en XLM con el fin de estructurar los textos e incorporar los metadatos extraídos del HTLM y añadir nuevos metadatos que enriquecen sustancialmente el etiquetado. Transformar los archivos en XML permite, como apuntan Serrat $\&$ Martínez (2012: 68):

[...] describir con precisión la estructura de los documentos, anotar información sobre los participantes en la situación comunicativa y facilitar el procesamiento del corpus en fases posteriores.

La estructura de nuestro etiquetado está basada en las características técnicas de los subtítulos, de modo que podemos analizar de forma cuantitativa los 
parámetros técnicos de la subtitulación. Con ese fin, se genera un XML al que se le añade la siguiente información (metadatos) en tres niveles: texto, subtítulo y línea. Por tanto, del archivo SRT que hemos descargado tenemos la siguiente información: un nombre de archivo que lo identifica, el tipo de programa, la categoría, la URL del vídeo, la fecha de publicación, el número de subtítulos de ese vídeo y su duración, además del número de subtítulos de una línea o de dos líneas. De igual modo, añadimos información del subtítulo con datos relacionados con el tiempo de entrada y salida, la duración, el número de caracteres por segundo y la pausa entre subtítulos. Ahora nuestro texto raíz, ese archivo SRT que contenía información limitada en texto plano, pasa a ser un XML con información de gran interés para un estudio de estas características.

Conviene resaltar que también se ha añadido información lingüística a los textos (mediante procesos de tokenización, lematización y análisis morfosintáctico) obtenida con TreeTagger (Schmid 1994), se ha segmentado el texto en oraciones con NLTK (Bird et al. 2009), y, finalmente, se han alineado los subtítulos entre ambos idiomas (inglés y español).

El corpus EMPAC se ha indexado con el IMS Open Corpus Workbench (CWB). Este dispone de una interfaz gráfica (CQPweb; Hardie, 2012) que permite utilizar el procesador CQP a través del navegador y realizar búsquedas complejas además de explorar el corpus de forma automatizada. La riqueza de los datos, metadatos y anotaciones con el que hemos dotado al corpus permite analizar distintos parámetros inherentes a la subtitulación.

Por último, puesto que parte de nuestro estudio se basa en un análisis cuantitativo de datos numéricos hemos transformado los XML en archivos de Excel, lo que nos permite realizar una descripción estadística del corpus y llevar a cabo análisis bivariable y tablas cruzadas con el programa de análisis estadístico IBM SPSS.

\section{Resultados del análisis de la accesibilidad a los contenidos audiovisuales en la web}

A continuación, mostramos los resultados del análisis inicial, es decir, el grado de accesibilidad de los contenidos audiovisuales en la web del EuroparlTV a partir de las tres fases descritas en el apartado 6. 
Los resultados obtenidos en la primera fase del análisis (ficha 1) se muestran en la siguiente tabla. Los vídeos están agrupados por idiomas: para inglés (EN) y para español (ES). La última columna corresponde al porcentaje de vídeos que incorpora subtítulos en cada versión.

Tabla 7: Resultados de análisis inicial. Fase 1

\begin{tabular}{|c|c|c|c|}
\hline Idioma & Subtítulos & Vídeos & Porcentaje \\
\hline \multirow{2}{*}{ EN } & NO & 169 & $4,18 \%$ \\
\cline { 2 - 4 } & SÍ & 3878 & $95,82 \%$ \\
\hline \multirow{2}{*}{ ES } & NO & 71 & $1,76 \%$ \\
\cline { 2 - 4 } & SÍ & 3974 & $98,24 \%$ \\
\hline
\end{tabular}

Como podemos comprobar casi la totalidad de los vídeos incorporan subtítulos, en especial la versión en español, con un 98,24 \% de los vídeos emitidos con subtítulos.

En la segunda fase de nuestro análisis (ficha 2) determinamos qué tipo de subtítulos (SPS o convencional) incorpora cada vídeo. La herramienta CQPweb nos permite crear búsquedas para localizar todos los paréntesis y corchetes del corpus y la información en mayúsculas (está es la forma en la que se muestra la información sonora relevante en SPS) de forma automática. El resultado que obtenemos en el análisis tanto en la versión en español como en inglés es que, de los escasos paréntesis y corchetes detectados en el total de los subtítulos, ninguno de ellos contenía información sonora relevante. Tampoco la información en mayúsculas.

Por último, con el objetivo de determinar si los vídeos incorporaban audiodescripción (ficha 3) inspeccionamos la web y no encontramos ninguna información para activar la audiodescripción. Acto seguido, nos adentramos en el código fuente en el que se alojan los vídeos y tampoco localizamos ninguna etiqueta ni información adicional sobre recursos de audiodescripción. Constatamos por duplicado que, en los HTML asociados a EuroparlTV, no 
hay ningún tipo de información (ni visible ni incrustada en código) en forma de enlaces o elementos de audio.

Por tanto, tras realizar la evaluación de la accesibilidad en las tres fases en las que dividimos el análisis inicial, podemos afirmar que, si bien casi todos los vídeos incorporan subtítulos, especialmente en el caso de español, ninguno incorpora información sonora relevante. En cuanto a la audiodescripción concluimos que ningún vídeo es accesible para personas ciegas y con discapacidad visual.

\section{Resultados del análisis del parámetro velocidad de lectura}

Con el objetivo de realizar un estudio pormenorizado y obtener una aproximación más realista sobre la evolución de la velocidad de lectura en los subtítulos que componen nuestro corpus, realizamos un análisis bivariable de años y la variable analizada.

10.1 Variable número de caracteres por segundo: análisis por años

El análisis bivariable de años y cps nos permite comparar, según los tres valores propuestos, la cantidad de subtítulos con ese número de cps y rastrear su comportamiento a lo largo del tiempo. El valor 15 cps recoge los subtítulos con hasta 15 cps, el valor 20 cps, en naranja, los subtítulos con 16 cps hasta $20 \mathrm{cps}$, mientras que el valor $\leq 21$ da cuenta de los subtítulos con velocidades de 21 cps y más. Comenzamos mostrando los resultados del análisis en EMPAC_EN. 


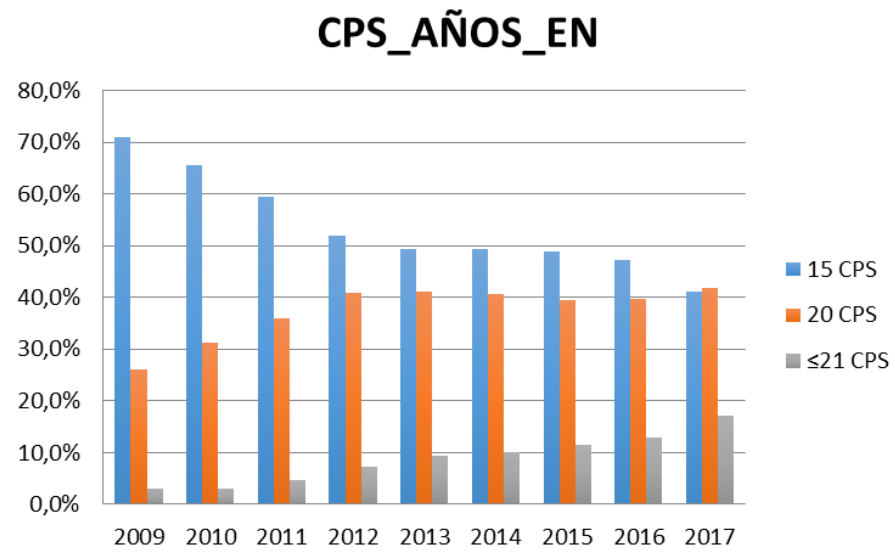

Figura 1: Resultado del análisis de cps por años en EMPAC_EN.

El valor 15 cps es descendente, mientras que el de 21 cps es ascendente, y aunque el valor 20 cps aumente hasta 2012 en los siguientes años se mantiene relativamente estable.

Asimismo, los datos muestran cómo en 2009 el $71 \%$ de los subtítulos cumplía con la norma de 15 cps; sin embargo, a partir de ese punto se produce un descenso paulatino hasta alcanzar un 41,1 \% en 2017. En el valor $\leq$ 21 cps vemos la misma tendencia, pero a la inversa, aunque el cambio no es tan acusado y pasamos del $3 \%$ al 17,2 \%, sin olvidar que en un corpus de las dimensiones que hemos descrito, el 17,2 \% no es una cantidad desdeñable. Y, por último, el valor 20 cps no sigue este mismo tipo de evolución ascendente/ descendente. Si bien durante los primeros años, hasta 2012, sí se produce un incremento, en los siguientes años la cifra se mantiene relativamente estable.

Al igual que en el caso anterior, en EMPAC_ES, se observa una tendencia al aumento de la velocidad de lectura de los subtítulos. Sin embargo, mientras que el número de subtítulos con velocidades de lectura de hasta 15 cps en 2009 es del 75 \%, en 2017 disminuye al 30\%. Con todo, quizás el dato más significativo es que, en 2017, el número de subtítulos con el valor $\leq 21$ cps es el más abultado de entre los tres valores y tan sólo el 30\% de los subtítulos tiene una velocidad de 15 cps. Recogemos toda esta información en la siguiente gráfica. 


\section{CPS_AÑOS_ES}

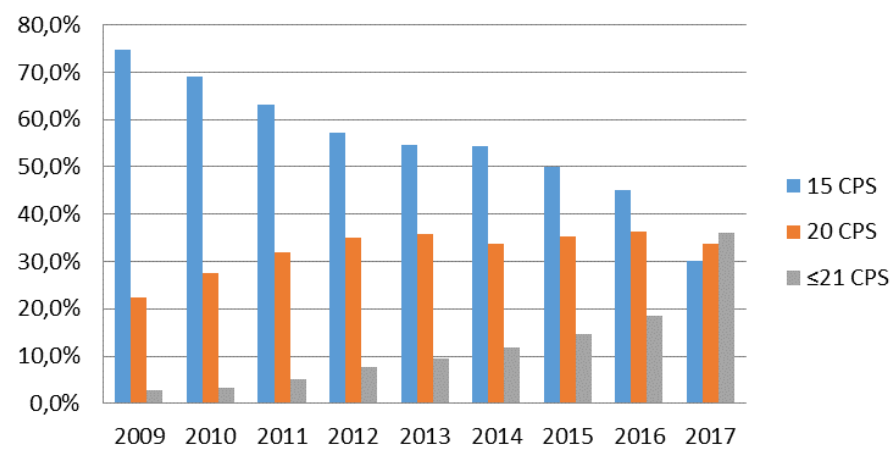

Figura 2: Resultado del análisis de cps por años en EMPAC_ES.

Por otra parte, si comparamos los datos de los subtítulos en inglés y en español de 2017, vemos claramente que el aumento de la velocidad de lectura en los subtítulos en español es mucho más elevado que en inglés.

\section{CPS_2017_EN/ES}

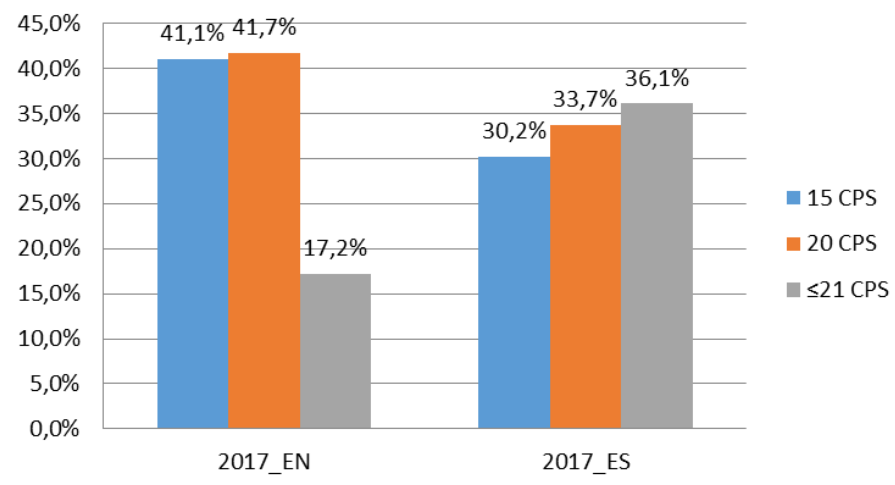

Figura 3: Resultado del análisis de cps en EMPAC_EN y EMPAC_ES. 
Esto nos lleva a pensar que es factible que, en 2017, exista la tendencia de generar subtítulos cada vez más verbatim y, por ende, más difíciles de leer y comprender.

Resumiendo, podemos afirmar que casi la mitad del corpus EMPAC supera los $15 \mathrm{cps}$, es decir, están por encima de lo que marca la norma UNE y DCMP. De hecho, es preocupante que en 2017 se aprecie un repunte significativo de subtítulos con 21 cps (subtítulos imposibles) o más en ambas versiones, lo que nos lleva a afirmar que son cada vez menos accesibles.

\subsection{Variable pausa entre subtítulos: análisis por años}

El análisis por años de esta variable muestra que, entre 2009 y 2015, el número de subtítulos sin pausa era extraordinariamente bajo; sin embargo, en 2016 se observa un incremento significativo respecto a los años anteriores. Pero es en 2017 cuando se produce un cambio radical en la tendencia en ambas versiones y más de la mitad de los subtítulos tienen Pausa 0 , es decir, no hay pausa alguna entre ellos.

En la tabla de datos que mostramos a continuación, podemos observar cómo en EMPAC_EN pasamos de un 1,7\% de subtítulos sin pausa en 2009 a un $68,1 \%$ en 2017. Por su parte, el valor de más de 16 milésimas es homogéneo, posiblemente debido al tipo de textos audiovisuales (textos institucionales) que conforman nuestro corpus. Por otra parte, como podemos apreciar en esta visualización minuciosa de los datos, los porcentajes son muy similares en ambas versiones. 
Tabla 8. Resultados del análisis de la pausa entre subtítulos en EMPAC_EN y EMPAC_ES

\begin{tabular}{|c|c|c|c|c|c|c|c|}
\hline EN & PAUSA 0 & $\leq 0,16$ & $\geq 0,16$ & ES & PAUSA 0 & $\leq 0,16$ & $\geq 0,16$ \\
\hline 2009 & $1,7 \%$ & $84,1 \%$ & $14,2 \%$ & 2009 & $1,2 \%$ & $85,7 \%$ & $13,0 \%$ \\
\hline 2010 & $1,8 \%$ & $81,8 \%$ & $16,4 \%$ & 2010 & $1,7 \%$ & $81,9 \%$ & $16,4 \%$ \\
\hline 2011 & $1,7 \%$ & $79,0 \%$ & $19,3 \%$ & 2011 & $1,6 \%$ & $78,6 \%$ & $19,8 \%$ \\
\hline 2012 & $1,6 \%$ & $78,0 \%$ & $20,4 \%$ & 2012 & $1,6 \%$ & $77,4 \%$ & $21,1 \%$ \\
\hline 2013 & $1,4 \%$ & $79,2 \%$ & $19,3 \%$ & 2013 & $1,4 \%$ & $78,8 \%$ & $19,8 \%$ \\
\hline 2014 & $1,7 \%$ & $78,6 \%$ & $19,6 \%$ & 2014 & $1,9 \%$ & $77,9 \%$ & $20,2 \%$ \\
\hline 2015 & $2,6 \%$ & $77,6 \%$ & $19,8 \%$ & 2015 & $2,6 \%$ & $77,6 \%$ & $19,8 \%$ \\
\hline 2016 & $8,8 \%$ & $72,9 \%$ & $18,3 \%$ & 2016 & $9,1 \%$ & $72,7 \%$ & $18,1 \%$ \\
\hline 2017 & $68,1 \%$ & $13,8 \%$ & $18,1 \%$ & 2017 & $64,3 \%$ & $16,5 \%$ & $19,2 \%$ \\
\hline
\end{tabular}

En suma, la variable pausa entre subtítulos indica que hasta 2016 casi la totalidad de los subtítulos respeta la norma de dejar un espacio de al menos 0,16 milésimas de segundo. Sin embargo, en 2016 se aprecia un cambio en la tendencia y es en 2017 cuando se produce un cambio radical y más del 60 $\%$ de los subtítulos no tienen pausa.

\subsection{Variable duración de los subtítulos: análisis por años}

En esta variable, mediante un análisis pormenorizado por años, observamos que en EMPAC_EN y EMPAC_ES el 90\% de los subtítulos permanece en pantalla entre 1 y 6 segundos, por lo que esta variable sí que está alineada con los valores propuestos en este estudio. Vemos a continuación los resultados que hemos obtenido. 
Tabla 9. Resultados del análisis de la duración de los subtítulos en EMPAC_EN y EMPAC_ES8

\begin{tabular}{|c|c|c|c|c|c|c|c|}
\hline EN & $>1 \mathrm{sg}$ & 1 y $6 \mathrm{sg}$ & $<6 \mathrm{sg}$ & ES & $>1 \mathrm{sg}$ & 1 y $6 \mathrm{sg}$ & $<6 \mathrm{sg}$ \\
\hline 2009 & $0,0 \%$ & $89,6 \%$ & $10,3 \%$ & 2009 & $0,0 \%$ & $90,3 \%$ & $9,6 \%$ \\
\hline 2010 & $0,0 \%$ & $92,6 \%$ & $7,4 \%$ & 2010 & $0,0 \%$ & $92,6 \%$ & $7,4 \%$ \\
\hline 2011 & $0,1 \%$ & $93,7 \%$ & $6,2 \%$ & 2011 & $0,1 \%$ & $93,8 \%$ & $6,1 \%$ \\
\hline 2012 & $0,2 \%$ & $94,4 \%$ & $5,4 \%$ & 2012 & $0,2 \%$ & $94,6 \%$ & $5,2 \%$ \\
\hline 2013 & $0,3 \%$ & $94,7 \%$ & $5,0 \%$ & 2013 & $0,3 \%$ & $94,8 \%$ & $4,8 \%$ \\
\hline 2014 & $0,0 \%$ & $93,3 \%$ & $6,7 \%$ & 2014 & $0,1 \%$ & $93,5 \%$ & $6,4 \%$ \\
\hline 2015 & $0,1 \%$ & $93,4 \%$ & $6,5 \%$ & 2015 & $0,1 \%$ & $93,4 \%$ & $6,5 \%$ \\
\hline 2016 & $0,1 \%$ & $93,2 \%$ & $6,7 \%$ & 2016 & $0,1 \%$ & $93,6 \%$ & $6,3 \%$ \\
\hline 2017 & $1,2 \%$ & $95,9 \%$ & $2,9 \%$ & 2017 & $1,1 \%$ & $96,2 \%$ & $2,8 \%$ \\
\hline
\end{tabular}

En EMPAC_EN apreciamos, por una parte, que en 2009 el 10,3 \% de los subtítulos permanecen en pantalla más de seis segundos, mientras que no hay subtítulos por debajo del segundo; sin embargo, en 2017, el número de subtítulos de menos de un segundo asciende hasta un 1,2\% y los de más de 6 segundos se reducen hasta un 2,9\%. Los datos en EMPAC_ES son muy similares, lo que parece lógico si tenemos en cuenta que el pautado de los subtítulos es igual para todas las lenguas. Se trabaja con una Master File por lo que todos los vídeos tienen el mismo número de subtítulos y la misma duración.

\section{Conclusiones}

La presente investigación nace de la inquietud por el impacto de la digitalización en la vida de las personas; sobre todo, en la de las personas con algún tipo de discapacidad sensorial. Sin embargo, no queremos dejar de señalar que esa inquietud y preocupación se extiende también a todas aquellas personas que no tienen conocimientos lingüísticos en otra lengua, en particular, de la lengua inglesa (hegemónica y globalizadora). Por tanto, en este trabajo hemos querido poner de relieve la importancia y el impacto de los avances tecnológicos en la sociedad actual, una sociedad en la que aquella persona 
que no tenga acceso a la información corre serio riesgo de quedar excluida y sin derecho a participar en ella de forma plena.

El material audiovisual alojado en EuroparlTV nos ha permitido, por una parte, estudiar un aspecto esencial de la subtitulación en el contexto institucional y, por otra, analizar el cumplimiento de los requisitos de accesibilidad de los contenidos audiovisuales establecidos por las Pautas de Accesibilidad al Contenido Web (WCAG) 2.0.

El análisis inicial demuestra que todos los subtítulos alojados en la web EuroparlTV son convencionales y no SPS. A su vez, hemos constatado que todos los vídeos que componen nuestro corpus carecen de audiodescripción, por ello afirmamos que los contenidos audiovisuales alojados en EuroparlTV no cumplen con los requisitos de accesibilidad establecidos por la WAI. Asimismo, apreciamos que las WCAG 2.0 se quedan en la superficie a la hora de ofrecer información para los desarrolladores de contenido web sobre cómo implementar y evaluar la SPS. Consideramos, por tanto, que sería necesario incluir indicaciones sobre los parámetros técnicos de la subtitulación en las WCAG, en concreto, en los documentos How to meet WCAG 2.0 y Understaning WCAG 2.0.

En otro orden de cosas, queremos destacar el esfuerzo que desde la UE se hace para procurar que, lingüísticamente, el contenido sea accesible, pero sirva este estudio para llamar la atención sobre los cambios producidos a partir de 2016. Los subtítulos a partir de esta fecha, si bien aparecen en todas las lenguas oficiales de la UE, no son necesariamente accesibles, como hemos constatado en la segunda parte de nuestro análisis. En él describimos en qué medida los parámetros técnicos de la subtitulación (velocidad de lectura) están presentes en EMPAC y la evolución de este parámetro (estudio diacrónico) en los valores asignados a cada variable. Por tanto, queda dibujado el panorama pasado y presente de la velocidad de lectura en los subtítulos que conforman nuestro corpus.

Entre 2009 y 2016 los porcentajes de cps son similares en EMPAC_EN y EMPAC_ES. Se observa una evolución progresiva hacia subtítulos más rápidos en ambas versiones; sin embargo, los subtítulos en inglés (hasta 2016) siempre son un poco más rápidos que los españoles.

En 2016 es el primer año en el que los subtítulos en español son ligeramente más exigentes que en inglés, pero es en 2017 cuando se produce 
un cambio sustancial, no solo respecto al resto de años sino también entre EMPAC_EN y EMPAC_ES. De hecho, solo un 30,2\% de los subtítulos en español respetan la norma de 15 cps y el 36,1\% tienen 21 cps o más. En EMPAC_EN, por su parte, si bien el porcentaje de subtítulos con 20 cps se ha doblado respecto a 2009 , todavía un $41,1 \%$ estarían en el valor normativo (15 cps), y el 17,2\% con 21 cps o más.

Esta evolución nos lleva a pensar que en 2009 los subtítulos en español se editaban más que los subtítulos en inglés que, por ser la lengua pivote de las Master File, tiende desde sus orígenes a estar más cerca de la transcripción verbatim que de la subtitulación. De hecho, por lo que hemos podido comprobar, la mayor parte de los subtítulos en inglés son transcripciones verbatim que, con el paso del tiempo, han aumentado la velocidad de lectura reduciendo el tiempo de permanencia en pantalla de los subtítulos. Sin embargo, en el caso de los subtítulos en español, estos sí están editados hasta 2016, pero a partir de septiembre de ese año cambia la forma de generar subtítulos y se asemejan más a una traducción convencional que a una subtitulación. No se sintetiza ni se tiene en cuenta el tiempo que es necesario para poder leer toda la información que aparece en pantalla, lo que los convierte en subtítulos imposibles o inaccesibles, tanto para las personas sordas como para las personas oyentes.

Por último, este estudio ha servido para presentar el corpus EMPAC, que será de acceso libre a través de la web https://empac-corpus.com. Esperamos que sea la base para futuras investigaciones que contribuyan a mejorar la accesibilidad a los contenidos audiovisuales en la web.

\section{Referencias bibliográficas}

AENOR. (2012) Subtitulado para personas sordas y personas con discapacidad auditiva, Norma UNE 153010:2012. Madrid: AENOR.

BIRD, Steven; Edward Loper \& Ewan Klein. (2009) Natural Language Processing with Python. Beiking et al.: O'Reilly Media Inc.

BBC. (2009) Online Subtitling Editorial Guidelines. Versión electrónica: <http:// www.bbc.co.uk/guidelines/futuremedia/accessibility/subtitling_guides/ online_sub_editorial_guidelines_vs1_1.pdf>

CASTELlS, Manuel. (2000) "Tecnologías de la información y desarrollo global." Estudios de política exterior 14, pp. 151-168. 
CAStro Roig, Xavier. (2004) "Solo ante el subtítulo: experiencias de un subtitulador.” En: Pereira Rodríguez, Ana María \& Lourdes Lorenzo García (coord.) 2001. Traducción subordinada II, El subtitulado: (inglés-español/gallego). Vigo. Servizo de Publicacións da Universidade de Vigo.

DCMP. (2009) Captioning Key. Guidelines and preferred techniques. Versión electrónica: <http://www.dcmp.org/captioningkey/about_c.html>

Delabastita, Dirk. (1989) "Translation and mass-communication: film and T.V. translation as evidence of cultural dynamics." Babel 35:4, pp. 193-218.

DíAz CinTAS, Jorge. (2001) La traducción audiovisual. El subtitulado. Salamanca: Almar.

DíAz CinTAS, Jorge. (2007) "Traducción audiovisual y accesibilidad." En: Jiménez Hurtado, Catalina (ed.) 2007. Traducción y accesibilidad: Subtitulación para sordos y audiodescripción para ciegos: nuevas modalidades de traducción audiovisual. Frankfurt: Peter Lang, pp. 9-23.

DíAz CinTAS, Jorge \& Aline Remael. (2007) Audiovisual Translation: Subtitling. Manchester: St. Jerome Publishing

Díaz Cintas, Jorge. (2009) New Trends in Audiovisual Translation. Búfalo: Multilingual Matters.

Díaz CinTAS, Jorge. (2012) "Los subtítulos y la subtitulación en clase de lengua extranjera." Abehache 3. Versión electrónica: <http://discovery.ucl. ac.uk/1451727/1/DiazCintas_abehache_12.pdf>

DíAZ CinTAS, Jorge; Aline Remael \& Pilar Orero (eds.) (2007) Media for All: Subtitling for the Deaf, Audio Description and Sign Language. Amsterdam \& New York: Rodopi.

EVERT, Stevan \& Andrew Hardie. (2011) "Twenty-first century Corpus Workbench: Updating a query architecture for the new millennium." Proceedings of the Corpus Linguistics 2011 conference. Birmingham: University of Birmingham. FuENTES-LuQUE, Adrián. (2015) "Institutional Audiovisual Translation: A (Shop) Window on the World." En: Baños Piñero Rocio \& Jorge Díaz Cintas (eds.) 2015. Audiovisual Translation in a Global Context. London. Palgrave Macmillan.

Gómez Hernández, Jose Antonio; Antonio Calderón Rehecho \& José Antonio Magna Wals (coord.) 2008. Brecha digital y nuevas alfabetizaciones. El papel de la biblioteca. Madrid: Biblioteca de la Universidad Complutense de Madrid. GonZÁlez-IGlesias, David. (2012) Desarrollo de una herramienta de análisis de los parámetros técnicos de los subtítulos y estudio diacrónico de series 
estadounidenses de televisión en DVD. Salamanca: Universidad de Salamanca. Tesis doctoral inédita.

HARDIE, Andrew. (2012) "CQPweb - combining power, flexibility and usability in a corpus analysis tool." International Journal of Corpus Linguistics 17:3, pp. 380-409.

ISO (2012) ISO/IEC 40500:2012. Information technology - W3C Web Content Accessibility Guidelines (WCAG) 2.0. Versión electrónica: <http://www.iso. org/iso/iso_catalogue/catalogue_tc/catalogue_detail.htm?csnumber=58625>

ITU (2003) "Declaración de Principios: Construir la Sociedad de la Información: un desafío global para el nuevo milenio." Versión electrónica: <https://www. itu.int/dms_pub/itu-s/md/03/wsis/doc/S03-WSIS-DOC-0004!!PDF-S.pdf>

IVARSSON, Jan \& Mary Carroll. (1998) Subtitling. Simrishamn: Transedit.

KARAMITROGLOU, Fotios. (1998) "A proposed set of subtitling norms in Europe." Translation Journal 2:2. Versión electrónica: <https://translationjournal.net/ journal/04stndrd.htm>

MAYORAL, Roberto. (2001a) Aspectos epistemológicos de la traducción. Castellón: Universitat Jaume I.

MAYORAL, Roberto. (2001b) "Campos de estudio y trabajo en traducción audiovisual." En: Duro Moreno, Miguel (coord.) 2001. La traducción para el doblaje y la subtitulación. Madrid: Cátedra, pp. 19-45.

ONU. (1948) Declaración Universal de Derechos Humanos. Asamblea General de Naciones Unidas. 10 de diciembre 1948, 217 A (III) Versión electrónica: <https://www.refworld.org.es/docid/47a080e32.html>

ORERO, Pilar. (2005) "La inclusión de la accesibilidad en comunicación audiovisual dentro de los estudios de traducción audiovisual." Quaderns. Revista de traducció 12, pp. 173-185.

ORREGO, David. (2013) "Avance de la traducción audiovisual: desde los inicios hasta la era digital." Mutatis Mutandis 6:2, pp. 297-320.

PEDERSEN, Jan. (2017) "The FAR model: assessing quality in interlingual subtitling." The Journal of Specialised Translation 28. Versión electrónica: <https:// www.jostrans.org/issue28/art_pedersen.php>

RemaEl, Aline. \& Joselia Neves (eds.) (2007) "A Tool for social integration? Audiovisual translation from different angles." Linguistica Antverpiensia, New Series 6, pp. 11-22.

ROMERO-FresCO, Pablo. (2019). Accessible Filmmaking: Integrating translation and accessibility into the filmmaking process. London: Routledge. 
SCHMID, Helmut. (1994) "Probabilistic Part-of-Speech Tagging Using Decision Trees." Proceedings of International Conference on New Methods in Language Processing. Manchester, UK.

Serrat Roozen, Iris \& José Manuel Martínez Martínez. (2012) "ECPC: el discurso parlamentario europeo desde la perspectiva de los estudios traductológicos de corpus." Linguamática 4:2, pp. 65-73.

Sponholz, Christine. (2003) Teaching Audiovisual Translation. Theoretical Aspects,

Market Requirements, University Training and Curriculum Development.

(Trabajo fin de carrera). Mainz: Johannes Gutenberg-Universität.

TAMAYO, Ana. (2015) Estudio descriptivo y experimental de la traducción en TV para niños sordos. Una propuesta alternativa. Universitat Jaume I.

Castellón. Tesis doctoral inédita. Versión electrónica: <https://www.tdx.cat/ handle/10803/353962>

VOCES Merayo, Ramón. (2010) Comunicación audiovisual sin barreras: Televisión pública. World Wide Web y Accesibilidad. Universitat Autònoma de Barcelona. Tesis doctoral inédita.

W3C. (2008) Web Content Accessibility Guidelines 2.0. Versión electrónica: <http:// www.w3.org/TR/WCAG20/>

W3C. (2014) How to meet WCAG 2.0. Versión electrónica: <https://www.w3.org/ WAI/WCAG21/quickref/?versions=2.0>

W3C. (2014) Understanding WCAG 2.0. Versión electrónica: <https://www. w3.org/WAI/GL/2016/WD-UNDERSTANDING-WCAG20-20160105/understanding-techniques.html\#understanding-techniques>

\section{BIONOTA / BIONOTE}

IRIS SERRAT ROOZEN es Doctora en Lenguas Aplicadas, Literatura y Traducción por la Universitat Jaume I. Actualmente es profesora asociada del Departamento de Filología Inglesa y Alemana de la Universidad de Valencia. Sus principales líneas de investigación son la traducción audiovisual, la accesibilidad, y el aprendizaje y la enseñanza de segundas lenguas.

IRIS SERRAT ROOZEN holds a PhD in Applied Languages, Literature and Translation. Currently, she is an associate lecturer in language teaching at the Department of English and German Studies at the Universitat de València. Her main research interests are audiovisual translation, accessibility, and second language learning and teaching. 